\title{
MID-INFRARED IMAGING OF THE TRANSITIONAL DISK OF HD 169142: MEASURING THE SIZE OF THE GAP*
}

\author{
M. Honda ${ }^{1}$, Koen MaAskant ${ }^{2,3}$, Y. K. OKamoto ${ }^{4}$, H. Kataza ${ }^{5}$, M. Fukagawa ${ }^{6}$, L. B. F. M. Waters ${ }^{2,7}$, \\ C. Dominik ${ }^{2,8}$, A. G. G. M. Tielens ${ }^{3}$, G. D. Mulders ${ }^{2,9}$, M. Min $^{10}$, T. Yamashita $^{11}$, T. FujiYoshi ${ }^{12}$, \\ T. MiYATA ${ }^{13}$, S. SAKo $^{13}$, I. SAKON ${ }^{14}$, H. FuJiWARA ${ }^{12}$, AND T. ONAKA ${ }^{14}$ \\ ${ }^{1}$ Department of Mathematics and Physics, Faculty of Science, Kanagawa University, 2946 Tsuchiya, Hiratsuka, Kanagawa 259-1293, Japan \\ 2 Astronomical Institute Anton Pannekoek, University of Amsterdam, P.O. Box 94249, 1090 GE Amsterdam, The Netherlands \\ ${ }^{3}$ Leiden Observatory, Leiden University, P.O. Box 9513, 2300 RA Leiden, The Netherlands \\ ${ }^{4}$ Institute of Astrophysics and Planetary Sciences, Faculty of Science, Ibaraki University, 2-1-1 Bunkyo, Mito, Ibaraki 310-8512, Japan \\ ${ }_{5}^{5}$ Department of Infrared Astrophysics, Institute of Space and Astronautical Science, Japan Aerospace Exploration Agency, \\ 3-1-1 Yoshinodai, Sagamihara, Kanagawa 229-8510, Japan \\ ${ }^{6}$ Department of Earth and Space Science, Graduate School of Science, Osaka University, 1-1 Machikaneyama, Toyonaka, Osaka 560-0043, Japan \\ ${ }^{7}$ SRON Netherlands Institute for Space Research, Sorbonnelaan 2, 3584 CA Utrecht, The Netherlands \\ ${ }^{8}$ Department of Astrophysics/IMAPP, Radboud University Nijmegen, P.O. Box 9010, 6500 GL Nijmegen, The Netherlands \\ ${ }^{9}$ SRON Netherlands Institute for Space Research, P.O. Box 800, 9700 AV Groningen, The Netherlands \\ ${ }^{10}$ Astronomical Institute Utrecht, Utrecht University, P.O. Box 80000, 3508 TA Utrecht, The Netherlands \\ ${ }^{11}$ National Astronomical Observatory of Japan, 2-21-1 Osawa, Mitaka, Tokyo 181-8588, Japan \\ ${ }^{12}$ Subaru Telescope, National Astronomical Observatory of Japan, 650 North A'ohoku Place, Hilo, HI 96720, USA \\ ${ }^{13}$ Institute of Astronomy, School of Science, University of Tokyo, 2-21-1 Osawa, Mitaka, Tokyo 181-0015, Japan \\ ${ }^{14}$ Department of Astronomy, Graduate School of Science, University of Tokyo, Bunkyo-ku, Tokyo 113-0033, Japan \\ Received 2011 December 19; accepted 2012 April 17; published 2012 June 5
}

\begin{abstract}
The disk around the Herbig Ae star HD 169142 was imaged and resolved at 18.8 and $24.5 \mu \mathrm{m}$ using Subaru/COMICS. We interpret the observations using a two-dimensional radiative transfer model and find evidence for the presence of a large gap. The mid-infrared images trace dust that is emitted at the onset of a strong rise in the spectral energy distribution (SED) at $20 \mu \mathrm{m}$, and are therefore very sensitive to the location and characteristics of the inner wall of the outer disk and its dust. We determine the location of the wall to be $23_{-5}^{+3} \mathrm{AU}$ from the star. An extra component of hot dust must exist close to the star. We find that a hydrostatic optically thick inner disk does not produce enough flux in the near-infrared, and an optically thin, geometrically thick component is our solution to fit the SED. Considering the recent findings of gaps and holes in a number of Herbig Ae/Be group I disks, we suggest that such disk structures may be common in group I sources. Classification as group I should be considered a strong case for classification as a transitional disk, though improved imaging surveys are needed to support this speculation.
\end{abstract}

Key words: circumstellar matter - protoplanetary disks - stars: pre-main sequence

\section{INTRODUCTION}

Transitional disks are a class of protoplanetary disks whose inner regions are devoid of small dust grains. These disks have been attracting attention recently, since such cleared inner regions or gaps may be related to ongoing planet formation. The presence of an inner hole/gap has been suggested for the disk around the Herbig Ae star HD 169142 by Grady et al. (2007) and Meeus et al. (2010), mostly based on analysis of the spectral energy distribution (SED). Both groups conclude that the steep rise in flux at $\sim 20 \mu \mathrm{m}$ in the SED reflects emission from the wall of the outer disk. The location of the wall (i.e., the inner edge of the outer disk) has not been determined conclusively-Meeus et al. (2010) used 20 AU while Grady et al. (2007) suggested 44 AU. Observations of HD 169142 at various wavelengths have so far not been able to constrain the inner cavity radius directly and accurately.

One of the limitations of SED modeling is the inability to locate and prove the existence of gaps in protoplanetary disks. For instance, gaps may not be revealed in the SED because the inner disk can partially obscure large parts of the outer disk (Acke et al. 2009) and gaps hidden under that shadow do

\footnotetext{
* Based on data collected at Subaru Telescope, which is operated by the National Astronomical Observatory of Japan.
}

not leave a fingerprint in the SED. Clever SED modeling can reproduce SEDs of truly transitional disks without the need for a gap. The transitional disk LkCa 15 has an outer disk starting at $46 \mathrm{AU}$ as seen at 1.4 and $2.8 \mathrm{~mm}$ wavelengths (Piétu et al. 2006). However, Isella et al. (2009) show that a model approach with a smooth distribution of material from a few stellar radii to about 240 AU can sometimes reproduce both the observed SED and the spatially resolved continuum emission at millimeter wavelengths for this object. Only from direct imaging is it possible to directly constrain the radial density structure of dust in a protoplanetary disk.

Dust in protoplanetary disks covers a temperature range from $\sim 1500 \mathrm{~K}$ at the inner dust sublimation radius to a few Kelvin in the outer parts of the disk. Direct mid-infrared (MIR) imaging at 18.8 and $24.5 \mu \mathrm{m}$ is most sensitive for $100-150 \mathrm{~K}$ blackbody dust, though strong contributions from dust elsewhere in the disk at lower and higher temperatures can also be present. If the disk has a very strong near-infrared (NIR) component such as HD 135344 (Meeus et al. 2001), the inner region may still be strongly represented in the center of the image at $18 \mu \mathrm{m}$. On the other hand, for disks with a larger gap or an inner hole, the image size will be a sensitive tracer for the location of the inner radius of the outer disk (Verhoeff et al. 2011).

In this paper, we present direct MIR imaging observations of HD 169142 at 18.8 and $24.5 \mu \mathrm{m}$, using the Cooled 
Table 1

Summary of Observations

\begin{tabular}{|c|c|c|c|c|c|}
\hline Object & Filter & $\begin{array}{l}\text { Date } \\
\text { (UT) }\end{array}$ & $\begin{array}{l}\text { Integ. Time (s) } \\
\text { Used/Total (\%) }\end{array}$ & Airmass & $\begin{array}{c}\text { Direct } \\
\text { FWHM }\end{array}$ \\
\hline$\delta \mathrm{Oph}$ & Q24.5 & 2004 Jul 11 & $195 / 243$ s $(80 \%)$ & $1.254-1.354$ & $0.628 \pm 0.007$ \\
\hline HD 169142 & Q24.5 & 2004 Jul 11 & $345 / 802$ s $(43 \%)$ & $1.541-1.542$ & $0^{\prime \prime} 680 \pm 0.034$ \\
\hline$\alpha$ Her & Q18.8 & 2004 Jul 12 & $83 / 83$ s $(100 \%)$ & $1.081-1.115$ & $0^{\prime \prime} 493 \pm 0.006$ \\
\hline HD 169142 & Q18.8 & 2004 Jul 12 & $215 / 360 \mathrm{~s}(60 \%)$ & $1.553-1.559$ & $0^{\prime \prime} 604 \pm 0.017$ \\
\hline
\end{tabular}

Mid-Infrared Camera and Spectrometer (COMICS) on the $8.2 \mathrm{~m}$ Subaru telescope. We confirm the result found by Mariñas et al. (2011) and find that the disk shows extended emission at $18 \mu \mathrm{m}$. In addition we find that the disk is also resolved at $24.5 \mu \mathrm{m}$. By constructing a radiative transfer disk model that fits both the SED and the imaging results, we find that the size of the source at MIR wavelengths is most naturally explained by a disk with a large inner gap, i.e., a transitional disk. We discuss the structure of the disk and the implications for its nature.

\section{OBSERVATIONS AND DATA REDUCTION}

Observations were conducted using COMICS (Kataza et al. 2000; Okamoto et al. 2003; Sako et al. 2003) on the $8.2 \mathrm{~m}$ Subaru Telescope on Mauna Kea, Hawaii. HD 169142 was observed using the Q24.5-NEW $(\lambda=24.5 \mu \mathrm{m}, \Delta \lambda=0.75 \mu \mathrm{m})$ and Q18.8 $(\lambda=18.8 \mu \mathrm{m}, \Delta \lambda=0.9 \mu \mathrm{m})$ filters. The plate scale of the COMICS camera was 0.13 arcsec pixel ${ }^{-1}$. The chopping throw was 10 arcsec and the position angle of the chopping direction was $0^{\circ}$. The chopping frequency was $0.45 \mathrm{~Hz}$. The total integration times for Q24.5 and Q18.8 observations were $802 \mathrm{~s}$ and $360 \mathrm{~s}$, respectively. Just before and after observing HD 169142, we took data from point spread function (PSF) reference and photometric standard stars. We used $\delta$ Oph for Q24.5 and $\alpha$ Her for Q18.8. The total integration times for the reference stars were $243 \mathrm{~s}$ and $83 \mathrm{~s}$, respectively. A summary of the observations is given in Table 1.

We processed the data using a shift-and-add method to improve the blurring caused by atmosphere, tracking errors, misregistration, etc. The imaging data consisted of $0.98 \mathrm{~s}$ onsource integration frames. First, the thermal background and the dark current signals were removed through the subtraction of the chopped pair frames. The object and the PSF star are bright enough to be recognized even in $0.98 \mathrm{~s}$ chop-subtracted frames, so we searched the centroid of the object. We then shifted the frames so as to align the centroid position and summed up the frames. However, we excluded frames which were blurred by atmospheric seeing. We also rejected frames whose radius of $78.4 \%$ encircled energy $\left(r_{78.4}\right)$ was larger than the threshold radius $\left(r_{c}\right)$ which covers $95 \%$ of the wavefronts whose Strehl ratio is 0.9 . These threshold values are determined as $r_{c}=1^{\prime \prime} .131$ at $18.8 \mu \mathrm{m}$ and $r_{c}=1^{\prime \prime} .482$ at $24.5 \mu \mathrm{m}$ by Monte Carlo simulation of the ideal unresolved point source. Due to this rejection of lower quality data, the effective integration time of HD 169142 was reduced to $345 \mathrm{~s}$ and $215 \mathrm{~s}$ for Q24.5 and Q18.8, respectively. The same procedure was applied to the PSF images taken before and after HD 169142, where the effective integration time became $195 \mathrm{~s}$ and $83 \mathrm{~s}$, respectively. Since HD 169142 shows marginal extension compared to the PSF, and the rejection criterion $r_{c}$ is determined for the ideal unresolved point source, frame rejection rates of HD 169142 are higher than those of PSF stars. This means that the effective rejection criterion is slightly more stringent for HD 169142 than for PSF stars, however, it does not overestimate the extension of HD 169142.

For flux calibration we used template spectra provided by Engelke et al. (2006). Using the standard stars observed over the two nights, an airmass correction was applied for Q24.5 photometry. For Q18.8 photometry, we could not find a significant airmass dependence during the night. A standard aperture photometry was applied and the resultant flux density of HD 169142 was $10.5 \pm 0.4 \mathrm{Jy}$ at $18.8 \mu \mathrm{m}$ and $13.0 \pm 0.5 \mathrm{Jy}$ at $24.5 \mu \mathrm{m}$. The final images of the HD 169142 and PSF stars are shown in Figure 1.

\section{RESULTS}

\subsection{Source Size of HD 169142 at 18.8 and $24.5 \mu \mathrm{m}$}

The azimuthally averaged radial brightness profiles of HD 169142 and the PSF stars at 18.8 and $24.5 \mu \mathrm{m}$ are shown in Figure 2. It is clear that HD 169142 is extended at these wavelengths. At $18.8 \mu \mathrm{m}$ the direct FWHM of the shift-andadded images of HD 169142 and of the PSF stars were 0".604 \pm 0.017 and $0^{\prime \prime} 493 \pm 0.006$, respectively. At $24.5 \mu \mathrm{m}$, the corresponding numbers are 0.'680 \pm 0!.034 and 0'.628 \pm 0.007 (see Table 1). It is surprising that the size of HD 169142 does not increase with wavelength. For a continuous flaring disk, one would expect the size of the image to scale roughly with the PSF (Meijer 2007). Our images therefore give a first indication that the radial density structure is not continuous. The FWHMs of the PSF references are comparable to the predicted value of the diffraction-limited performance of the telescope. As a rough estimate of the intrinsic source size of HD 169142, we applied the quadratic subtraction method as described by Mariñas et al. (2011). The derived source FWHMs were 0'349 $\pm 00^{\prime} 014$ at $18.8 \mu \mathrm{m}$ and $0^{\prime} .261 \pm 0^{\prime \prime} 025$ at $24.5 \mu \mathrm{m}$. Since Mariñas et al. (2011) observed the FWHM of this source to be 0'32 \pm 0 0'05 at $18.0 \mu \mathrm{m}$, our measurement at $18.8 \mu \mathrm{m}$ is in good agreement with their results within the uncertainties.

\section{MODELING}

\subsection{Observational Constraints}

Complementary views of the disk structure and the dust properties of HD 169142 are obtained by adopting photometric data at various wavelengths from the literature (van den Ancker et al. 1997; Cutri et al. 2003; Zacharias et al. 2004; Sylvester et al. 1996; Meeus et al. 2010) and MIR spectra from ISO (Meeus et al. 2001) and Spitzer/Infrared Spectrograph (IRS; Juhász et al. 2010). These data sets reflect the disk brightness as a function of wavelength, and are a result of the disk structure defined by the density distribution, composition, inclination, and inner and outer radius of the disk, and by the properties of the central star. An outer disk radius of $235 \mathrm{AU}$ is taken from Panić et al. (2008). An inner disk radius of $0.1 \mathrm{AU}$ is set at the location where the dust reaches $1500 \mathrm{~K}$ and starts to sublimate. With 

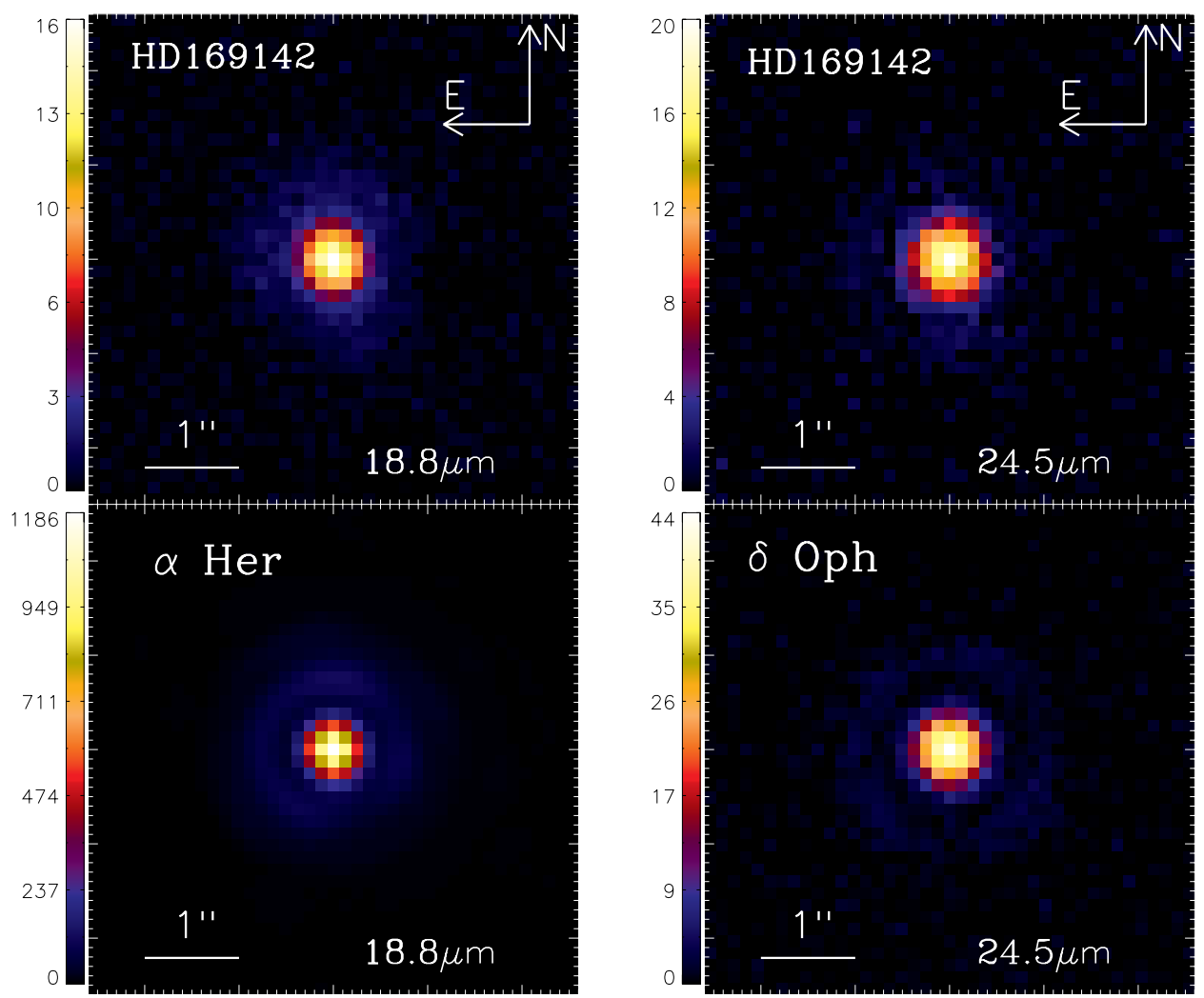

Figure 1. Shift-and-added image of HD 169142 (top) and the PSF stars (bottom) at 18.8 and $24.5 \mu \mathrm{m}$. The brightness unit is Jy arcsec ${ }^{-2}$ and is scaled from 0 to the peak value in the image. North is up and east is to the left.

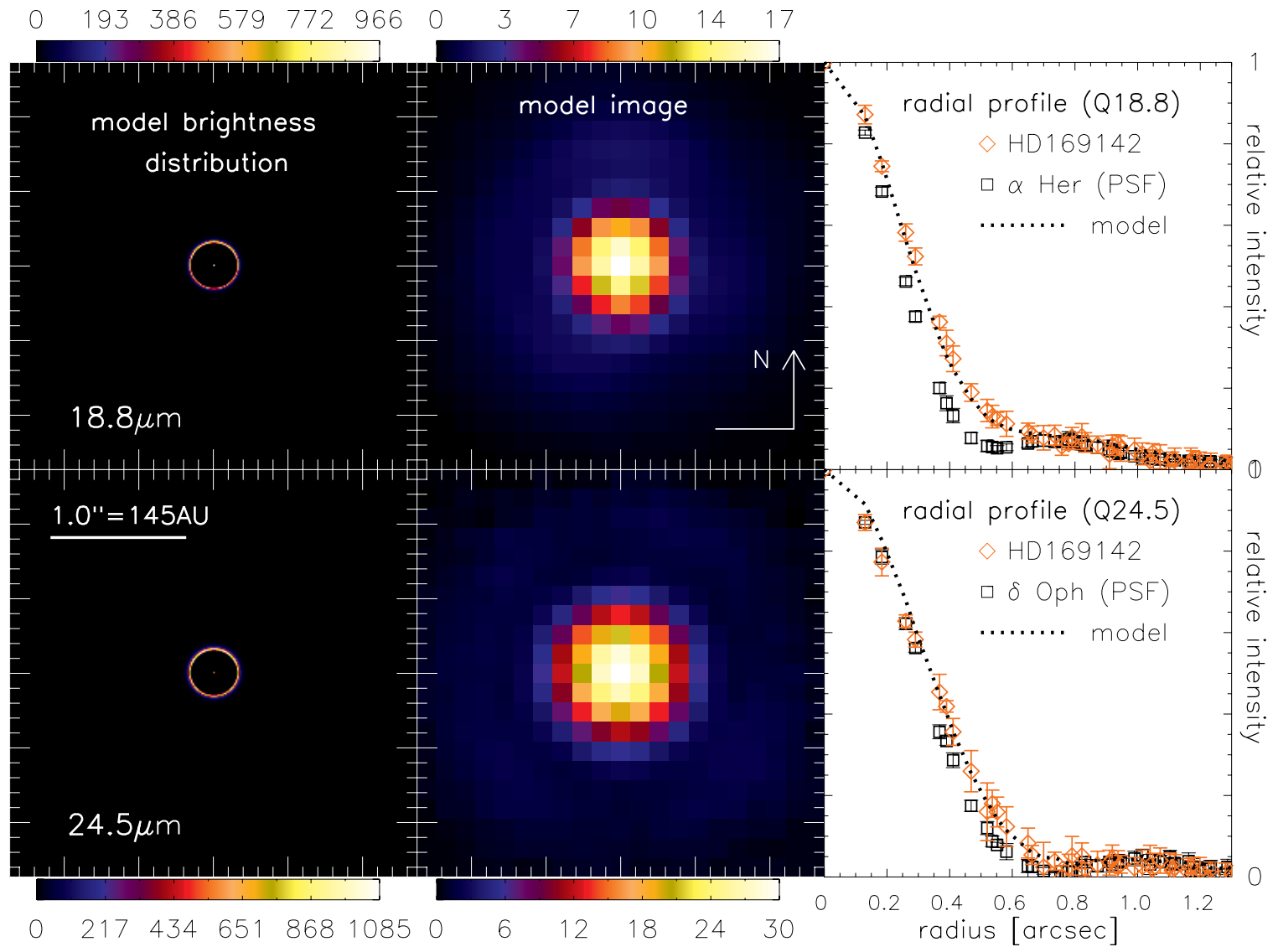

Figure 2. The left and middle panels show the model brightness distribution of the final model and model image after convolving by Subaru/COMICS PSF (middle), respectively, shown in Jy $\operatorname{arcsec}^{-2}$. The right panels represent the peak-normalized azimuthally averaged radial brightness profiles relative to the centroid of the image (right). The top panels are in the Q18.8 and the bottom panels are in the Q24.5 filters. In the radial profile plots (right), the observation of HD 169142 is shown by the red diamonds, and the corresponding PSF of the calibrators is shown by the black squares. The final model convolved with the PSF is shown by the black dotted line. 
Table 2

Parameters of HD 169142 System Used in our Best-fit Model

\begin{tabular}{|c|c|c|}
\hline Parameter & Value & Remarks \\
\hline Spectral type & $\mathrm{A} 5 \mathrm{Ve}$ & Dunkin et al. (1997) \\
\hline Extinction $A_{V}$ & $0.46 \pm 0.05$ & van den Ancker (1999) \\
\hline $\log g$ & 4.22 & van den Ancker (1999) \\
\hline Temperature & $8200 \mathrm{~K}$ & Dunkin et al. (1997) \\
\hline Distance & $145 \pm 15 \mathrm{pc}$ & de Zeeuw et al. (1999) \\
\hline Age & $6_{-3}^{+6} \mathrm{Myr}$ & Grady et al. (2007) \\
\hline Stellar luminosity & $15.33 \pm 2.17 L_{\odot}$ & van den Ancker (1999) \\
\hline Stellar mass & $2.28 \pm 0.23 M_{\odot}$ & van den Ancker (1999) \\
\hline Stellar radius & $1.94 \pm 0.14 R_{\odot}$ & van den Ancker (1999) \\
\hline Gas disk mass & $(0.16-3.0) \times 10^{-2} M_{\odot}$ & Panić et al. (2008) \\
\hline Dust disk mass & $4 \times 10^{-4} M_{\odot}$ & Fit to the submillimeter photometry \\
\hline Inclination & $13^{\circ}$ & Raman et al. (2006); Dent et al. (2005) \\
\hline Accretion rate & $\leqslant 10^{-9} M_{\odot} \mathrm{yr}^{-1}$ & Grady et al. (2007) \\
\hline$R_{\text {halo }}$ & $0.1-0.2 \mathrm{AU}$ & Geometrically high, optically thin component to fit the NIR \\
\hline$R_{\text {in }}$ & $23_{-5}^{+3} \mathrm{AU}$ & Fit to RBP of Subaru/COMICS data \\
\hline$R_{\text {out }}$ & $235 \mathrm{AU}$ & Panić et al. (2008) \\
\hline Surface density exponent & -1.0 & Hydrostatic equilibrium \\
\hline Particle size & $a=\{0.03 \mu \mathrm{m}, 1 \mathrm{~cm}\}$ & Power-law distribution of -3.5 \\
\hline Silicates & $70 \%$ & Similar to Mulders et al. (2011) \\
\hline Amorphous carbon & $30 \%$ & Zubko et al. (1996) \\
\hline$M_{\mathrm{PAH}}$ & $0.45 \times 10^{-7} M_{\odot}$ & Uniform PAH distribution \\
\hline$M_{\text {halo }}$ & $0.28 \times 10^{-10} M_{\odot}$ & Only carbon \\
\hline$M_{\text {disk }}$ & $0.3 \times 10^{-3} M_{\odot}$ & Mass of grains $a=\{0.03 \mu \mathrm{m}, 1 \mathrm{~cm}\}$ in the disk \\
\hline
\end{tabular}

an inclination of $13^{\circ}$ (Raman et al. 2006), the system is nearly pole-on and, therefore, deviations from axial symmetry caused by projection effects can be neglected in our images. The stellar spectrum is described by a Kurucz model with an effective temperature of $8200 \mathrm{~K}$ (Dunkin et al. 1997), luminosity of $15.3 L_{\odot}$ and extinction $A_{V}=0.46$ (van den Ancker 1999). The system is set at a distance of $145 \mathrm{pc}$ (de Zeeuw et al. 1999). All stellar and disk parameters used in this paper are shown in Table 2.

\subsection{Radiative Transfer Code MCMax}

We construct a geometrical model of the disk of HD 169142 and fit it to both the radial surface brightness profile (RBP) of our observed images and to the SED. For the model we use the radiative transfer code MCMax (Min et al. 2009), which is able to solve temperature and density structures in very optically thick circumstellar disks. This code has been compared to other radiative transfer codes by Pinte et al. (2009) and has been applied successfully in previous studies (e.g., Verhoeff et al. 2011; Mulders et al. 2011). We assume an axisymmetric dust distribution in which the optical properties of the grains are computed using a distribution of hollow spheres (Min et al. 2005). We consider all dust species in the disk to be in thermal contact, and calculate their respective opacities from the optical constants as if they were separate particles. Furthermore, we assume the gas temperature to be set by the dust temperature. We adopted grains with sizes according to the power law $f(a) \propto a^{-3.5}$. The power-law index is that of interstellar grains (Mathis et al. 1977) and is consistent with collisional fragmentation considerations (Hellyer 1970). Under the assumption that the system is in hydrostatic equilibrium, the vertical density distribution of the disk is solved by iterating the density and temperature structures until they become selfconsistent (e.g., Dullemond et al. 2007).

We assume a grain population consisting of $30 \%$ carbon and $70 \%$ silicates (we refer to Mulders et al. 2011 for references of the optical properties of this dust composition). The contribution of warm small $(<10 \mu \mathrm{m})$ amorphous silicate grains to the spectrum must be low, since the flux level at $10 \mu \mathrm{m}$ is very low compared to that at $20 \mu \mathrm{m}$. We model this change in flux level by replacing the amorphous silicate dust in the inner disk with dust with a higher continuum opacity. Here we use carbon, but other dust species such as metallic iron may give the same result. This treatment is consistent with that of van Boekel et al. (2004) who found that grain growth in the innermost regions proceeded further than in the outer disk regions. We have not attempted to fit the polycyclic aromatic hydrocarbon (PAH) features in detail, though we implement them into our model to fit the SED. Scattered light contribution is also included and the fraction of the scattered light to the total flux density in 18.8 and $24.5 \mu \mathrm{m}$ is $22 \%-26 \%$ in this model.

\subsection{The Best-fit Model}

We fit the SED as well as the 18.8 and $24.5 \mu \mathrm{m}$ images simultaneously by using the disk parameters shown in Table 2 . The RBPs and SED of our final best-fit model are shown by the purple dashed lines in Figures 2 and 3. A schematic sketch is given in Figure 4. In this section we will first discuss the geometrical characteristics of the location of the gap and the outer disk properties. Thereafter, we discuss the constraints we find to the inner disk structure of the best-fitting model.

\subsubsection{Fitting the SED}

Previous SED modeling by Meeus et al. (2010) and Grady et al. (2007) suggested a gap in the disk with an outer radius of 20 and $44 \mathrm{AU}$, respectively. We confirm and improve on this result by our test modeling for HD 169142: disks without a gap fail to fit the SED, despite various attempts within the physically reasonably parameter space. So already the SED fitting gives a strong indication for the presence of a gap (confirming the results by Meeus et al. 2010; Grady et al. 2007, even though its size is not at all well constrained from the SED alone. We incorporate this result and include a gap in our model. The effect of the discontinuity in the radial density structure is that a large fraction of the stellar light now falls on the vertical 


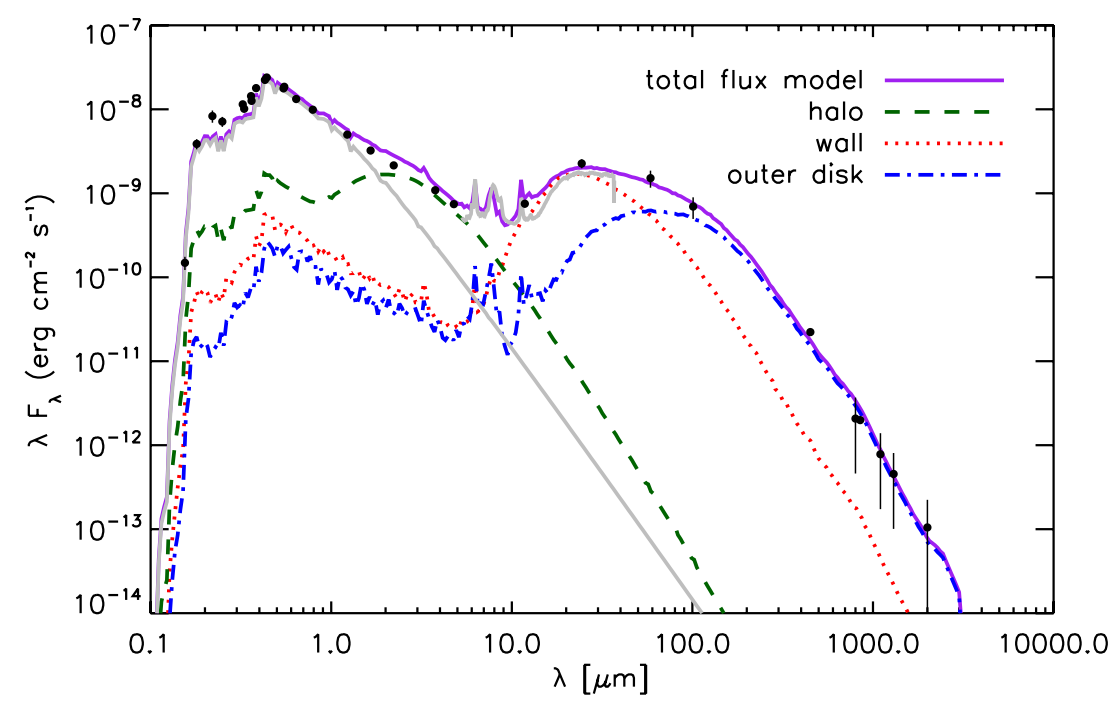

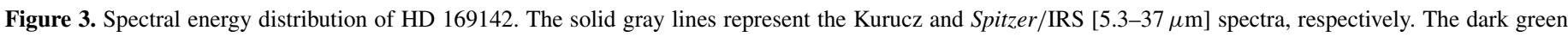

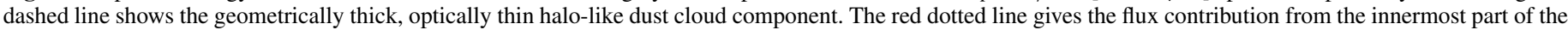

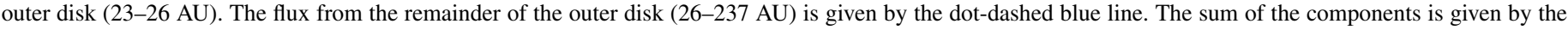
purple solid line. It is clear that at both 18.8 and $24.5 \mu \mathrm{m}$, the SED is dominated by flux coming from the inner edge of the outer disk.

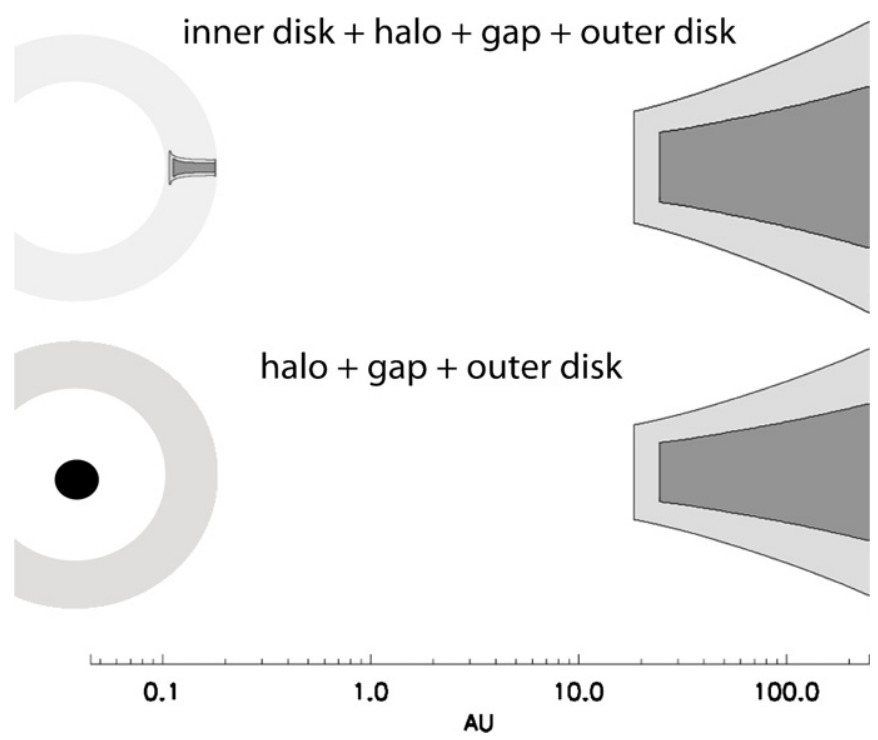

Figure 4. Sketch of our final model with two possible structures for the inner regions. Top: the model with a flat inner disk, an optically thin halo, and an outer disk. Bottom: the model with a denser but still optically thin halo, and an outer disk in which the scale height of the dust is decreased. Both geometries give an equally good fit to the SED and the images. Although we cannot distinguish between these models with our data set, we choose the bottom model as our final model described in the text.

wall at the inner edge of the outer disk. Emission from this wall is clearly distinguishable in the SED (Figure 3, illustrated by the red dotted line). In addition, Figure 3 shows that the flux contribution from the inner edge (between 23 and $26 \mathrm{AU}$ ) dominates at 18.8 and $24.5 \mu \mathrm{m}$ and that flux contributions from the inner regions and from the outer disk are negligible at these wavelengths. Therefore, our Subaru/COMICS images at 18.8 and $24.5 \mu \mathrm{m}$ are sensitive tracers of the radius where the outer disk starts. Another insight to help understand the dominance of the wall is given by the radial density and temperature plots of the outer disk of this model in Figure 5. The dotted lines show that the densest regions are largely optically thick. Therefore, the temperature of the disk mid-plane decreases rapidly as a function of radius. Thus 18.8 and $24.5 \mu \mathrm{m}$ photons are mostly produced at the inner few $\mathrm{AU}$ radii of the outer disk.

The radius where the outer disk starts is not well constrained by SED modeling, as the disk structure is degenerate by uncertainties of many parameters, such as the stellar temperature and luminosity, the interstellar extinction, the grain size population of the dust, the vertical density structure, and so on. Only by fitting our model to the resolved MIR observations can we unambiguously confirm the existence of a gap and constrain the location of the wall of the outer disk.

\subsubsection{Fitting the MIR Images, Constraining the Location of the Wall}

After convolving our best-fit model with the PSFs of the calibrators, we find that the imaging data are best fitted with an inner edge of the outer disk of $23_{-5}^{+3}$ AU (see Figure 2). The uncertainty on this radius is determined by fitting the uncertainty of the azimuthally averaged flux level at $\pm 1 \sigma$. This result is consistent with a wall radius of 20 AU (Meeus et al. 2010) but excludes the model with a wall at 44 AU (Grady et al. 2007).

We have performed a small parameter study where we have examined the robustness of our final model to fit the RBPs at MIR wavelengths. We have varied the following parameters: the surface density power law between $\{-0.5,-1.5\}$, the grain size distribution power law $\{-2.5,-4.5\}$, the dust settling factor $\{0.5,1\}$, the stellar temperature $\{8000 \mathrm{~K}, 8500 \mathrm{~K}\}$ and luminosity $\left\{13 L_{\odot}, 17 L_{\odot}\right\}$, and the distance $\{130 \mathrm{pc}, 160 \mathrm{pc}\}$. While the RBPs vary only slightly in this parameter study (all within the $1 \sigma$ error of the measurements), the SED fit becomes significantly worse. We conclude that the radius of the wall is the only free parameter which significantly influences the broadness of the RBP. Therefore, our fit to the RBP is robust in constraining the radius of the inner edge of the outer disk at $23_{-5}^{+3} \mathrm{AU}$.

\subsubsection{The Inner Disk}

In this section we explain in more detail the implications for the inner disk structure. Modeling shows that an optically thick hydrostatic inner disk does not produce enough flux to fit the SED. Scaling up the vertical height of the inner disk would not be a solution because if the inner disk is higher 

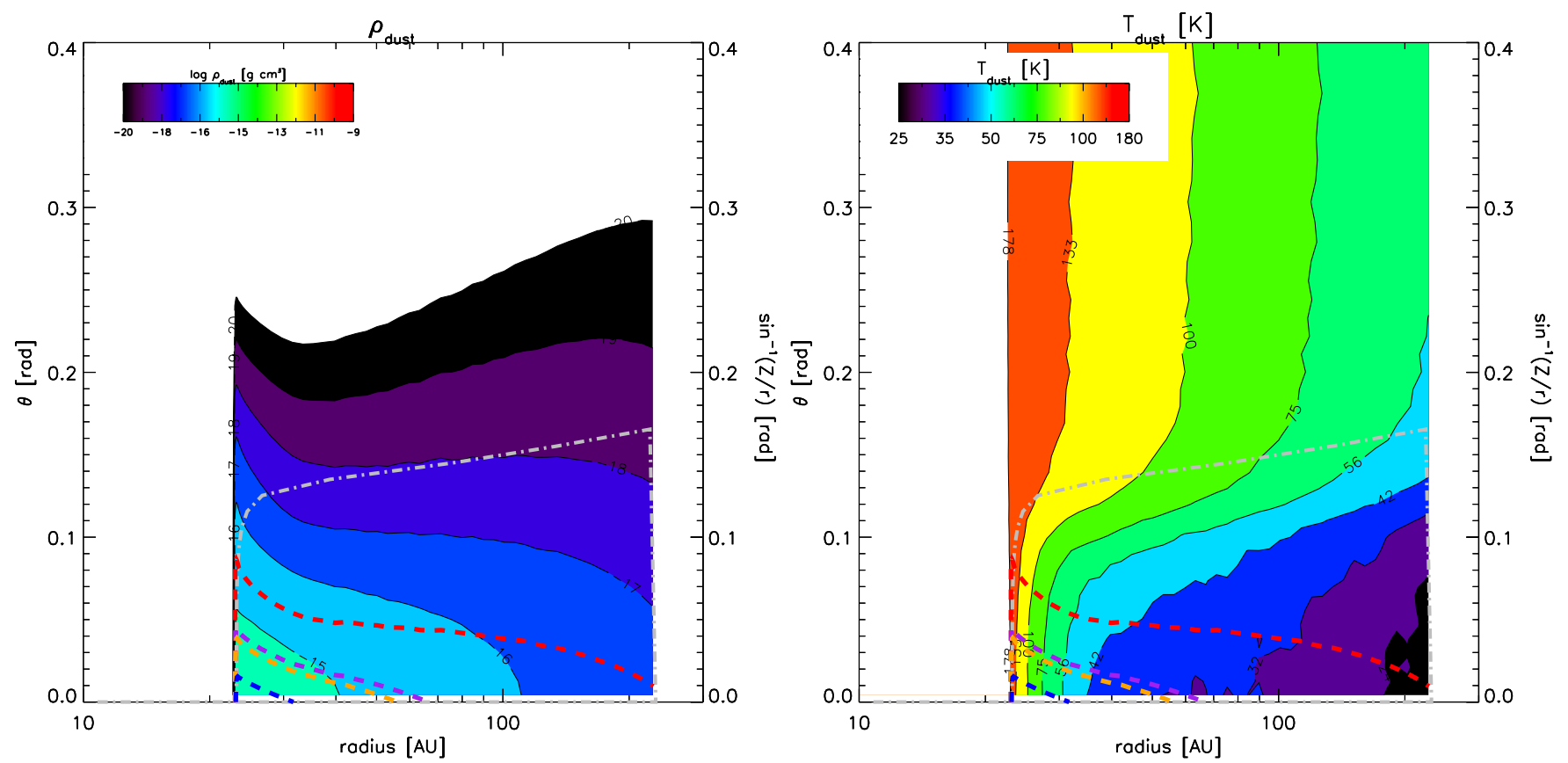

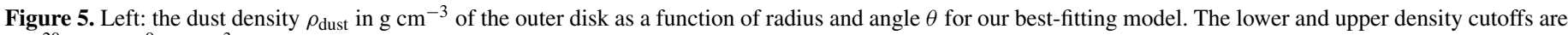

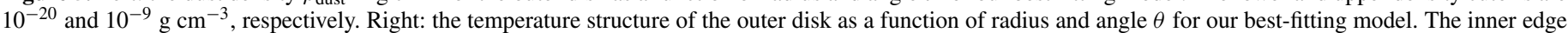

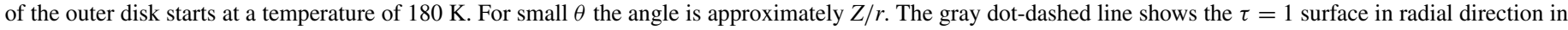

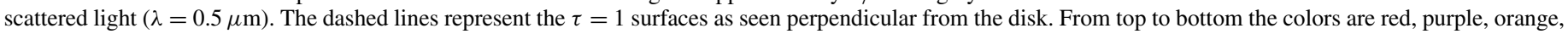
and blue and represent the wavelengths $0.5,18.8,24.5$, and $70 \mu \mathrm{m}$.

than $Z / r \sim 0.1$ it covers the outer disk for more than $\sim 50 \%$ in direct and scattered light. This inner disk then casts too large a shadow on the outer disk to be able to reproduce the SED at wavelengths longer than $\sim 10 \mu \mathrm{m}$. To solve this problem we include a geometrically thick, optically thin halo-like dust cloud in the inner regions $(<0.5 \mathrm{AU}$, see Figure 5$)$ to fit the SED in the NIR and to avoid a shadowing effect on the outer disk. The physical origin is not certain at this moment, though other observational studies show that an optically thin component close to the star provides a good fit as well (Grady et al. 2007; Benisty et al. 2011; Verhoeff et al. 2011; Mulders et al. 2010). Furthermore, fitting the Herbig stars' median SED also requires such a halo (Mulders \& Dominik 2012). Further high spatial resolution observations such as infrared interferometry will be necessary to reveal the inner disk structure.

If there is no shadowing effect from the inner disk, the wall of the outer disk is too luminous. To fit the SED again, we can either settle the grains in the outer disk by scaling down the dust-density scale height by a factor of 0.6 or we can scale down the abundance of small $(<1 \mu \mathrm{m})$ grains by one order of magnitude. These adjustments are consistent with dust settling and grain growth (Dullemond \& Dominik 2005); however, it is uncertain to what extent these effects are present in the disk. The influence of other effects, like dynamical scattering of larger dust grains may also play a role in the vertical grain size distribution. Alternatively, we can combine a halo-like dust cloud and a flat inner disk and consequently perform no settling or removal of small dust grains. So we conclude that there are two possible inner disk geometries (as illustrated in the schematic in Figure 4) which give an equally good fit to the SED and the RBP.

Without an optically thick inner disk, the RBP is still reproduced with the inner radius of the outer disk at $23_{-5}^{+3} \mathrm{AU}$. The final model can be interpreted as an inner halo-like dust cloud (Krijt \& Dominik 2011) and a gas-rich outer disk structure, and so is truly a transitional disk.

\section{DISCUSSION}

\subsection{Is the Inner Hole/Gap Structure Common for Meeus's Group I Sources?}

We have shown observational evidence for the presence of an inner hole or gap in the HD 169142 disk. A similar structure is also indicated for other Herbig Ae/Be stars such as AB Aur (Honda et al. 2010), HD 142527 (Fukagawa et al. 2006; Fujiwara et al. 2006; Verhoeff et al. 2011), HD 135344 (Brown et al. 2009), HD 36112 (Isella et al. 2010), and HD 100546 (Bouwman et al. 2003; Benisty et al. 2010; Mulders et al. 2011). All of these objects have been classified as group I according to the definition introduced by Meeus et al. (2001). Since the strong far-infrared excess is a characteristic of group I sources, we speculate that this component may come from the emission from the inner edge of the outer disk. Consequently, we suggest that the inner hole or gap structure may be common for group I sources. If this is true, the hypothesis that group I flared disks evolve into group II flat disks due to dust sedimentation should be reconsidered, because the difference between groups I and II is not only the degree of disk flaring or grain growth, but also the presence of cleared inner regions (holes and gaps) in the disk.

As we have shown in the above analysis, the spatial extent of a Herbig Ae/Be disk at MIR wavelengths, often described by an FWHM, is strongly influenced by the position and temperature of the wall. Previous studies showed that group I sources are likely to be more extended than group II sources in the MIR wavelengths, and suggested that the disk geometry (flaring or flat disk) may play an important role in the thermal structure and MIR emission of the disk (Leinert et al. 2004; Liu et al. 2007; Mariñas et al. 2011). Our $24.5 \mu \mathrm{m}$ imaging survey of Herbig Ae/Be stars with Subaru/COMICS (M. Honda et al. 2012, in preparation) also confirms that many group I sources are extended. However, their spatial extent shows great diversity, from marginally to remarkably extended. Such diversity in the 
spatial extent in the MIR can be understood in terms of the distance, the inner disk structure, and the location of the wall of the outer disk. In fact, the temperature of the wall both in the AB Aur and HD 142527 systems appears to be relatively cool $(70-100 \mathrm{~K})$ and its wall radius also tends to be at some distance away from the central star (100 AU in AB Aur; 170 AU in HD 142527; Fujiwara et al. 2006). The wall in HD 169142 is at a smaller distance (23 AU), thus the spatial extent is not so large compared to that of AB Aur and HD 142527. We suggest that not only the geometry (flared or flat) of the disk, but also the inner gap and the wall-like inner edge of the outer disk are important for understanding the spatial extent of Herbig Ae/Be disks in the MIR.

\subsection{Origin of the Inner Hole of the HD 169142 Disk}

Gaps seem to be a common characteristic of group I flaring disks (Honda et al. 2010; Fukagawa et al. 2006; Fujiwara et al. 2006; Verhoeff et al. 2011; Brown et al. 2009; Isella et al. 2010; Bouwman et al. 2003; Benisty et al. 2010; Mulders et al. 2011). An inner hole or gap in the disk has been explained by several mechanisms, including (1) photoevaporation of the disk (Alexander et al. 2006), (2) geometric shadowing (Dullemond \& Dominik 2004), (3) grain growth in the inner disk causing a lower dust opacity zone (van Boekel et al. 2005), and (4) the presence of another body in the disk that dynamically creates a gap and decouples the inner disk from the outer disk (Augereau \& Papaloizou 2004). We prefer solution (4) since this also provides a natural explanation for an inner halo-like dust cloud in the inner disk. The presence of a halo likely requires some dynamical interactions with planetary bodies since new generations of dust can then be created in situ up to large disk heights (Krijt \& Dominik 2011). Grain growth cannot explain the presence of a gap and a wall. Furthermore, our test models show that geometric shadowing (i.e., varying scale heights, surface density power laws) of a part of the disk at several AU cannot reproduce the SED and the RBP of the images. We recognize that planetary companions have not yet been detected in the transitional disk around HD 169142, though recent direct detection of the planets around A-type stars (e.g., HR8799; Marois et al. 2008, $\beta$ Pic; Lagrange et al. 2010) lends support to such a hypothesis. Thus we suggest that the HD 169142 system is an excellent candidate to look for newly formed planets in the protoplanetary disk.

\section{CONCLUSIONS}

We arrive at the following conclusions.

1. HD 169142 is extended at MIR wavelengths with the Subaru/COMICS instrument. The derived source sizes from quadratic PSF reference subtraction are $0^{\prime \prime} .349 \pm 0.014$ at $18.8 \mu \mathrm{m}$ and $0^{\prime \prime} .261 \pm 0.025$ at $24.5 \mu \mathrm{m}$, respectively.

2. The observed sizes as well as the steep rise of the SED near $20 \mu \mathrm{m}$ require a disk model with an inner hole the size of $23_{-5}^{+3}$ AU, making HD 169142 a transitional disk.

3. HD 169142 is one of the growing number of Herbig Ae disks that are classified as group I sources and that also require the presence of a large inner gap. It appears that many, if not all, group I sources may be strong candidates for classification as transitional disks.

We are grateful to all of the staff members of the Subaru Telescope. We also thank Ms. Hitomi Kobayashi and Dr. Yuji Ikeda at Kyoto-Nijikoubou Co., Ltd. K.M. is supported by a grant from the Netherlands Research School for Astronomy (NOVA). This research was partially supported by KAKENHI (Grantin-Aid for Young Scientists B: 21740141) by the Ministry of Education, Culture, Sports, Science and Technology (MEXT) of Japan.

\section{REFERENCES}

Acke, B., Min, M., van den Ancker, M. E., et al. 2009, A\&A, 502, L17 Alexander, R. D., Clarke, C. J., \& Pringle, J. E. 2006, MNRAS, 369, 229 Augereau, J. C., \& Papaloizou, J. C. B. 2004, A\&A, 414, 1153 Benisty, M., Renard, S., Natta, A., et al. 2011, A\&A, 531, A84 Benisty, M., Tatulli, E., Ménard, F., \& Swain, M. R. 2010, A\&A, 511, A75 Bouwman, J., de Koter, A., Dominik, C., \& Waters, L. B. F. M. 2003, A\&A, 401, 577

Brown, J. M., Blake, G. A., Qi, C., et al. 2009, ApJ, 704, 496

Cutri, R. M., Skrutskie, M. F., van Dyk, S., et al. 2003, VizieR Online Data Catalog, 2246, 0

Dent, W. R. F., Greaves, J. S., \& Coulson, I. M. 2005, MNRAS, 359, 663 de Zeeuw, P. T., Hoogerwerf, R., de Bruijne, J. H. J., Brown, A. G. A., \& Blaauw, A. 1999, AJ, 117, 354

Dullemond, C. P., \& Dominik, C. 2004, A\&A, 417, 159

Dullemond, C. P., \& Dominik, C. 2005, A\&A, 434, 971

Dullemond, C. P., Henning, T., Visser, R., et al. 2007, A\&A, 473, 457

Dunkin, S. K., Barlow, M. J., \& Ryan, S. G. 1997, MNRAS, 286, 604

Engelke, C. W., Price, S. D., \& Kraemer, K. E. 2006, AJ, 132, 1445

Fujiwara, H., Honda, M., Kataza, H., et al. 2006, ApJ, 644, L133

Fukagawa, M., Tamura, M., Itoh, Y., et al. 2006, ApJ, 636, L153

Grady, C. A., Schneider, G., Hamaguchi, K., et al. 2007, ApJ, 665, 1391

Hellyer, B. 1970, MNRAS, 148, 383

Honda, M., Inoue, A. K., Okamoto, Y. K., et al. 2010, ApJ, 718, L199

Isella, A., Carpenter, J. M., \& Sargent, A. I. 2009, ApJ, 701, 260

Isella, A., Natta, A., Wilner, D., Carpenter, J. M., \& Testi, L. 2010, ApJ, 725, 1735

Juhász, A., Bouwman, J., Henning, Th., et al. 2010, ApJ, 721, 431

Kataza, H., Okamoto, Y., Takubo, S., et al. 2000, Proc. SPIE, 4008, 1144

Krijt, S., \& Dominik, C. 2011, A\&A, 531, A80

Lagrange, A.-M., Bonnefoy, M., Chauvin, G., et al. 2010, Science, 329, 57

Leinert, C., van Boekel, R., Waters, L. B. F. M., et al. 2004, A\&A, 423, 537

Liu, W. M., Hinz, P. M., Meyer, M. R., et al. 2007, ApJ, 658, 1164

Mariñas, N., Telesco, C. M., Fisher, R. S., \& Packham, C. 2011, ApJ, 737, 57

Marois, C., Macintosh, B., Barman, T., et al. 2008, Science, 322, 1348

Mathis, J. S., Rumpl, W., \& Nordsieck, K. H. 1977, ApJ, 217, 425

Meeus, G., Pinte, C., Woitke, P., et al. 2010, A\&A, 518, L124

Meeus, G., Waters, L. B. F. M., Bouwman, J., et al. 2001, A\&A, 365, 476

Meijer, J. 2007, PhD thesis, Astronomical Institute "Anton Pannekoek", University of Amsterdam

Min, M., Dullemond, C. P., Dominik, C., de Koter, A., \& Hovenier, J. W. 2009, A\&A, 497, 155

Min, M., Hovenier, J. W., \& de Koter, A. 2005, A\&A, 432, 909

Mulders, G. D., \& Dominik, C. 2012, A\&A, 539, A9

Mulders, G. D., Dominik, C., \& Min, M. 2010, A\&A, 512, A11

Mulders, G. D., Waters, L. B. F. M., Dominik, C., et al. 2011, A\&A, 531, A93

Okamoto, Y. K., Kataza, H., Yamashita, T., et al. 2003, Proc. SPIE, 4841, 169

Panić, O., Hogerheijde, M. R., Wilner, D., \& Qi, C. 2008, A\&A, 491, 219

Piétu, V., Dutrey, A., Guilloteau, S., Chapillon, E., \& Pety, J. 2006, A\&A, 460, L43

Pinte, C., Harries, T. J., Min, M., et al. 2009, A\&A, 498, 967

Raman, A., Lisanti, M., Wilner, D. J., Qi, C., \& Hogerheijde, M. 2006, AJ, 131, 2290

Sako, S., Okamoto, Y. K., Kataza, H., et al. 2003, PASP, 115, 1407

Sylvester, R. J., Skinner, C. J., Barlow, M. J., \& Mannings, V. 1996, MNRAS, 279,915

van Boekel, R., Min, M., Leinert, C., et al. 2004, Nature, 432, 479

van Boekel, R., Min, M., Waters, L. B. F. M., et al. 2005, A\&A, 437, 189

van den Ancker, M. 1999, PhD thesis, Astronomical Institute "Anton Pannekoek", University of Amsterdam

van den Ancker, M. E., The, P. S., Tjin A Djie, H. R. E., et al. 1997, A\&A, 324, L33

Verhoeff, A. P., Min, M., Pantin, E., et al. 2011, A\&A, 528, A91

Zacharias, N., Urban, S. E., Zacharias, M. I., et al. 2004, AJ, 127, 3043

Zubko, V. G., Mennella, V., Colangeli, L., \& Bussoletti, E. 1996, MNRAS, 282, 1321 